Background Ankyloglossia is a developmental anomaly causing restricted tongue mobility. Posterior types are usually identified latest and least commonly. Currently, there are no standardised national/international guidelines for diagnosis. This case series aims to highlight the importance of early diagnosis to reduce cases identified only following significant morbidity.

Methods Over a 2 year period, consecutive patients diagnosed with posterior ankyloglossia were identified.

Results Of the 15 patients identified, mean age at diagnosis was 24 days (range 4-42). 8(53\%) had regained their birthweight however, for $3(38 \%)$ of these, weight gain was slow/inadequate. The remaining $7(47 \%)$ had lost weight with a mean weight loss of $8.54 \%$ (range 2.56-16.06). 13(86\%) were exclusively breastfed, $1(7 \%)$ both breast- and formula-fed, and 1(7\%) formula-fed. Presenting features included poor latch (60\%), weight loss (47\%), sore nipples (40\%), irritability (40\%), poor weight gain $(20 \%)$, increased feed duration (20\%), and lethargy (20\%). $9(60 \%)$ were diagnosed by breastfeeding co-ordinators, $4(26 \%)$ by community midwives, and $1(7 \%)$ each by a paediatrician and neonatal nurse. All patients underwent a frenotomy following which both weight gain and feeding improved in $11(73 \%)$. Behavioural improvements were noted in 8(53\%). 11(73\%) mothers felt their baby's symptoms had improved. All of the 6 mothers who initially described symptoms of their own reported improvement.

Conclusion For many infants, posterior ankyloglossia is often detected only once feeding has deteriorated enough to result in significantly poor weight gain or weight loss. To prevent this and other morbidities shown in this small case series, a standardised assessment tool may be a useful method to facilitate earlier diagnosis and improve clinical practice.

\section{PO-0983 VIROLOGY ASSCOIATED WITH LUNG CONSOLIDATION IN INFANTS AND CHILDREN WITH ACUTE BRONCHIOLITIS}

${ }^{1}$ A Alhammadi, ${ }^{2} \mathrm{M}$ Hendaus, ${ }^{2} \mathrm{M}$ Khalifa, ${ }^{2} \mathrm{E}$ Muneer. ${ }^{1}$ Pediatrics, Weill Cornell Medical College and Hamad Medical Corporation (HMC), Doha, Qatar; ${ }^{2}$ Pediatrics, Hamad Medical Corporation (HMC), Doha, Qatar

\subsection{6/archdischild-2014-307384.1600}

Background Bronchiolitis, a lower respiratory tract infection that primarily affects the small airways (bronchioles), is a common cause of illness and hospitalisation in infants and young children. Although several Studies suggest that radiographs in children with typical bronchiolitis have limited value, chest $\mathrm{x}$ ray still performed on routine basis. There is limited data regarding which viral-associated bronchiolitis has the highest rate of consolidation on a Chest Radiograph.

Aim The purpose of our study is to determine which virus inducing bronchiolitis has the highest rate of consolidation of a chest radiograph.

Methods A retrospective and descriptive study was conducted at Hamad Medical Corporation (HMC).

Infants and children ages 0 to 18 months hospitalised in our paediatric unit with acute bronchiolitis from October 2010 to March 2013 were included in the study. The following data were collected: age at diagnosis, sex, direct fluorescent antibody (DFA) and results of chest radiograph.

Results The study comprised of 838 infants, median age 3.6 months, and boys constituted $60 \%$ of total infants. 606 infants and children had a routine chest radiograph done in the paediatric emergency centre prior to admission. $\mathrm{n}=226,37.3 \%$, showed normal findings on chest radiographs, while $\mathrm{n}=380$, $62.7 \%$ showed consolidations. 70 chest radiographs (18.4\%) with consolidation were attributed to infants and children with bronchiolitis and negative DFA.

The results of positive DFA associated with consolidation on chest radiograph were as follow:

Respiratory Syncythial Virus (RSV) 161, 42.4\%; rhinovirus 68, 17.9\%; Human metapneumovirus ( $h M P V)$ 25, 6.6\%; parainfulenza virus (type1) 3, 0.8\%; parainfluenza virus (type 2) 2, $0.5 \%$; parainfluenza virus (type 3$) 15,3.9 \%$; parainfluenza virus (type 4) 4, 1.1\%; coronavirus 11, 2.9\%; adenovirus 10,2.6\%; enterovirus 3, 0.8\%; bocavirus 5, 1.3\%;H1N1 2, 0.5\%; Influenzavirus B 1,0.3\%. There was no statistically significant difference relating chest consolidation with DFA status, $\mathrm{p}=0.773$

Conclusions Bronchiolitis can be triggered by a diversity of respiratory viruses that appear similar on a chest radiograph; therefore, chest imaging is not routinely required in the initial management of bronchiolitis unless the diagnosis is uncertain.

\section{PO-0984 ASTHMA: A DIAGNOSTIC DILEMMA}

${ }^{1} \mathrm{M}$ Lakhanpaul, ${ }^{2} \mathrm{D}$ Bird, ${ }^{3} \mathrm{~L}$ Culley, ${ }^{4} \mathrm{~N}$ Hudson, ${ }^{5} \mathrm{~N}$ Robertson, ${ }^{6} \mathrm{~N}$ Johal, ${ }^{7} \mathrm{M}$ McFeeters, ${ }^{8} \mathrm{C}$ Hamlyn-Williams, ${ }^{9} \mathrm{MRD}$ Johnson. ${ }^{1}$ General and Adolescent Paediatric Unit, UCL Institute of Child Health, London, UK; ${ }^{2}$ Medical and Social Care Education, University of Leicester, Leicester, UK; ${ }^{3}$ Health and Life Sciences, de Montfort University, Leicester, UK; ${ }^{4}$ School of Applied Social Sciences, de Montfort University, Leicester, UK; ${ }^{5}$ School of Psychology, University of Leicester, Leicester, UK; ${ }^{6} N / A, N / A$, Leicester, UK; ${ }^{7}$ School of Nursing and Midwifery, de Montfort University, Leicester, UK; ${ }^{8} \mathrm{UCL}$ Institute of Child Health, General and Adolescent Paediatric Unit, London, UK; ${ }^{9}$ Mary Seacole Research Centre, de Montfort University, London, UK

\subsection{6/archdischild-2014-307384.1601}

Asthma is one of the most common chronic conditions with 1.1 million children experiencing asthma in their childhood. Much of the related morbidity is due to poor management, particularly the under use of preventative medicine.

This was a collaborative participatory study aimed at identifying where along the asthma pathway resources needed to be focused to improve asthma management. Interviews and focus groups were used to explore barriers to optimal asthma management with communities, children, families and healthcare professionals (HCPs). Key themes were drawn from the data, prioritised and translated into an intervention.

Diagnosis was identified as the key priority and one that all parents/carers felt needed to be addressed first, although it was considered a low priority to HCP. For parents there was confusion surrounding the diagnostic process, and the label of asthma itself. The diagnostic process also raised concerns, with some HCPs being reluctant to diagnose or suggesting that some children may be 'too young to diagnose'. Parents and carers reported problems with delays in treatment following a diagnosis, and inconsistent information being provided at the point of diagnosis. To improve the diagnostic process, a multifaceted, integrated intervention programme was developed.

This study highlighted that 'getting a diagnosis' was a priority. The disparity in priorities between HCPs and families around 'getting a diagnosis' emphasises the importance of working collaboratively with families as well as HCPs to ensure that key priorities, for service users and providers, are understood and addressed appropriately.

Funded by the NIHR HS and DR Programme (09/2001/19). 